\title{
Human Carcinogenesis: The Role of Age and Gender
}

\author{
DOUG DIX \\ Department of Health Science and Nursing, University of Hartford, West Hartford, CT, U.S.A.
}

\begin{abstract}
Background/Aim: To identify the reason for age and gender differences in cancer risk. Patients and Methods: Age-standardized incidence rates for 17 cancer types were compared between genders in 50 populations. For each cancer type, the female/male rate ratio was listed in fixed order of population. Correlation coefficients were calculated between these lists in all pairwise combinations. For each population, the female/male rate ratio was listed in fixed order of cancer. Correlation coefficients were calculated between lists in all pairwise combinations. Results: Only four pairwise combinations for cancer type gave a correlation coefficient greater than 0.700. For each population, the lowest correlation coefficient was 0.950 . Conclusion: The reason for the differences in risk of cancer varies with each type of cancer, but remains fixed in all populations. It is suspected that species-specific genes control stem cell telomere dynamics in a fixed strategy at rates that vary among tissues and between genders.
\end{abstract}

For all cancer types that occur after infancy, incidence rates change with age in three phases: A period of low risk followed by a period of increasing risk followed by a plateau or decline in risk (1-3). The final phase, 'Clemmesen's hook,' was originally suspected of being an artifact of faulty data for the very elderly $(4,5)$. This is now known not to be the case $(6,7)$. It was also suspected of being a cohort phenomenon in cross-sectional data (8). But the 'hook' remained in cancer types with opposing temporal trends, making this artifact unlikely (9). Careful analysis of the second phase shows the increase in cancer risk with age to be at a constantly decreasing rate, thus making the 'hook' a necessary consequence (1-3). We are left with an inescapable conclusion: For all cancer types studied, risk increases beyond some critical age at a continuously decreasing rate, and then plateaus or begins to decline.

Correspondence to: Doug Dix, Department of Health Science and Nursing, University of Hartford, West Hartford, CT 06117, U.S.A. Tel: +1 8602431116, Fax: +1 8607685706, e-mail: Dix@hartford.edu

Key Words: Carcinogenesis, aging, gender, cancer incidence.
The critical age varies according to the tissue of tumor origin. For some, that age is in early childhood, as in acute lymphoblastic leukemia; for others, it is in adolescence, as in osteogenic sarcoma, or in early adult years, as in testicular cancer. For most tissues of tumor origin, the critical age is over 40 years. But for all cancer types, the pattern is the same: An increase in risk at a continuously decreasing rate followed by a plateau or decline in risk at older ages. This common pattern speaks volumes about the mechanism of carcinogenesis. Firstly, it suggests that the mechanism is fundamentally the same in all tissues. Secondly, it eliminates chance and the environment as the principal causes of cancer. If either chance or the environment were the principal cause of cancer $(10,11)$, risk would increase with age at a continuously increasing rate because mutations accumulate. Thirdly, it forces us to focus on the aging process and the normal genes that control that process as the principal cause of cancer (12-14).

Although mutations in proto-oncogenes and tumorsuppressor genes are commonly recognized as principal causes of cancer, the question remains as to what causes these mutations. Twin studies have eliminated segregating genes (15), which leaves only species-specific genes as reasonable suspects. Aging genes are species-specific (16). It is the purpose of this article to present data suggesting that they are likely the principal causes of carcinogenic mutations.

\section{Materials and Methods}

Age-standardized (according to the world standard) cancer incidence rates (ASRs: new cases/100,000 people/year) were obtained from a reliable source for cancer diagnosed between 2008 and 2012 (17). Data were analyzed only from registries without unusual caveats and reporting more than 100 cases of a given cancer. The following 50 populations fulfilled these criteria for most cancer types: Australia, Australia New South Wales, Australia Queensland, Austria, Brazil Curitiba, Bulgaria, Canada British Columbia, California Black, California White, China Beijing, China Hangzhou City, China Hong Kong, China Shanghai City, Croatia, Czech Republic, Denmark, Georgia (US) Black, Georgia (US) White, Germany Bavaria, Germany Lower Saxony, Greater Poland, Ireland, Israel, Italy Milan, Italy Turin, Japan Aichi, Japan Hiroshima, Japan Osaka, New York State Black, New York State 
White, New Zealand, North Carolina Black, North Carolina White, Norway, Poland Lower Silesia, Puerto Rico, Republic of Korea, Republic of Korea Seoul, Slovakia, Spain Basque Country, UK, UK England, UK Scotland, UK Wales, Ukraine, Uruguay, USA, USA Black, USA White, US Surveillance, Epidemiology, and End Results (SEER).

For each population, ASRs were analyzed in males and females separately for melanoma, myeloma, Hodgkin's lymphoma (HL), non-HL, and cancer of the mouth, esophagus, stomach, colon, liver, pancreas, breast, bronchus, kidney, brain, thyroid, larynx, and small intestine unless fewer than 100 cases were reported for a particular cancer type. Fewer than 100 cases were reported for males or females for the following types of cancer in the following populations: Laryngeal in Australia Queensland; mouth, esophageal, breast, thyroid, myeloma, laryngeal and small intestinal in Brazil; mouth, breast, and melanoma in California Black; breast and HL in China Beijing; laryngeal and HL in China Hangzhou; breast in China Hong Kong; breast, laryngeal, and HL in China Shanghai; mouth, breast, and melanoma in Georgia Black; breast and small intestinal in Greater Poland; breast and laryngeal in Italy Turin; laryngeal in Japan Aichi; breast, melanoma, laryngeal, and HL in Japan Hiroshima; breast and melanoma in New York State Black; mouth, breast, and melanoma in North Carolina Black; breast and small intestinal in Poland Lower Silesia; breast in Puerto Rico; breast and laryngeal in Republic of Korea Seoul; breast and laryngeal in Slovakia; breast and small intestinal in Spain Basque Country; breast in UK Wales; and breast and small intestinal in Uruguay.

Ratios of ASRs for females to those of males along with percentile distributions of ASRs and ratios and the Pearson correlation coefficient ( $\mathrm{r}$ ) between rates and ratios were calculated. The significance of correlation coefficients was calculated by the equation: $t=(r) \sqrt{(}\left(n-2 / 1-r^{2}\right)$. Coefficients were considered significant when $p<0.05$. Variation about the median $\left(\operatorname{Var}_{\text {med }}\right)$ was estimated by calculating the percentage of the median that was occupied by the 5 th to 95 th percentile range: $\operatorname{Var}_{\text {med }}=95$ th percentile -5 th percentile (100)/median.

Comparative statistics for females and males were obtained for life-expectancy (LE), gross national income (GNI), suicide risk (18), adult mortality rate (AMR) (19), infant mortality rate (IMR) and under-five mortality rate (U5MR) $(20,21)$ for Australia, Austria, Brazil, Bulgaria, Canada, China, Croatia, Czech Republic, Denmark, Germany, Ireland, Israel, Italy, Japan, New Zealand, Norway, Poland, Republic of Korea, Slovakia, Spain, UK, Ukraine, USA, and Uruguay, and for smoking rates for the above populations except Brazil, Canada, Croatia, Germany, Italy, and Poland (22).

\section{Results}

Tables I-III show percentiles and $\mathrm{Var}_{\text {med }}$ for male and female ASR and for the ratio of female/male ASR. In each table, cancer types are listed in order of median value of cancer type.

For each cancer type, ASR values were listed separately for females and males in order of population as follows: Puerto Rico, Uruguay, Canada British Columbia, USA, USA White, USA Black, China Beijing, China Hong Kong, Israel, Japan Osaka, Korea, Austria, Croatia, Czech Republic, Denmark, Germany Bavaria, Ireland, Italy Milan, Norway,
Table I. Male age standardized rate (ASR) percentiles and variation about the median (Varmed).

\begin{tabular}{lcccrr}
\hline & $\begin{array}{c}\text { Number of } \\
\text { populations }\end{array}$ & \multicolumn{3}{l}{ ASR percentile } & Var $_{\text {med }}$ \\
\hline Cancer & & $5 \%$ & $50 \%$ & $95 \%$ & \\
Bronchial & 50 & 30.5 & 43.8 & 57.4 & 61 \\
Colon & 50 & 14.2 & 24.4 & 30.6 & 67 \\
Melanoma & 45 & 0.50 & 12.1 & 39.8 & 325 \\
Kidney & 50 & 6.4 & 11.4 & 16.2 & 86 \\
Non-Hodgkin's & 50 & 4.8 & 11.1 & 15.3 & 95 \\
Stomach & 50 & 5.3 & 9.7 & 56.5 & 528 \\
Pancreatic & 50 & 5.6 & 8.5 & 11.1 & 65 \\
Liver & 50 & 3.3 & 7.8 & 24.8 & 276 \\
Brain & 50 & 2.9 & 6.3 & 7.4 & 71 \\
Esophageal & 49 & 3.0 & 5.6 & 11.3 & 148 \\
Laryngeal & 41 & 2.4 & 4.3 & 9.0 & 154 \\
Myeloma & 49 & 1.4 & 4.0 & 9.7 & 208 \\
Thyroid & 49 & 1.5 & 3.1 & 7.8 & 203 \\
Hodgkin's & 45 & 0.70 & 2.6 & 3.4 & 104 \\
Mouth & 45 & 0.90 & 1.9 & 3.1 & 116 \\
Small intestinal & 45 & 0.70 & 1.4 & 2.7 & 143 \\
Breast & 33 & 0.27 & 0.70 & 1.1 & 119 \\
\hline
\end{tabular}

Greater Poland, Spain Basque, Ukraine, UK, Australia, New Zealand, Brazil Curitiba, US SEER, China Shanghai, Japan Aichi, Korea Seoul, Bulgaria, Germany Lower Saxony, Poland Lower Silesia, Slovakia, UK England, UK Scotland, UK Wales, Australia New South Wales, Italy Turin, California White, California Black, Georgia White, Georgia Black, New York State White, New York State Black, North Carolina White, North Carolina Black, China Hangzhou, Japan Hiroshima, and Australia Queensland. Table IV provides the coefficients for correlation between these lists. Cancer types with $r>0.84$ for female versus male ASR had female/male $V r_{m e d}<65$. Except for cancer of the larynx, cancer types with $\mathrm{r}<0.75$ for female versus male ASR had female/male $\operatorname{Var}_{\text {med }}>64$.

For each cancer type, the ASR values for males were listed in order of population as described above. Correlation coefficients were calculated for the 17 cancer types in all pairwise combinations. Of the 136 coefficients, 64 were found to be significant. Of these 64 , only 23 were found to represent strong correlation $(> \pm 0.500)$. Of these 23,10 were greater than \pm 0.600 , but only three were highly strongly correlated $(> \pm 0.700)$ : stomach versus $\mathrm{HL}=-0.747$, stomach versus liver $=0.799$, and small intestine versus myeloma $=0.854$.

The ASR values for females were then also listed and pairwise analyzed as described above. Of the 136 coefficients, 90 were significant. Of these 90,47 were strongly correlated, with 25 being greater than \pm 0.600 , and nine being highly strongly correlated: kidney versus laryngeal $=0.705$, liver versus $\mathrm{HL}=-0.731$, stomach versus 
Table II. Female age standardized rate (ASR) percentiles and variation about the median (Varmed).

\begin{tabular}{lcccrr}
\hline \multirow{2}{*}{ Cancer } & & \multicolumn{3}{c}{ ASR percentile } & \multirow{2}{*}{ Var $_{\text {med }}$} \\
\cline { 3 - 4 } & $\begin{array}{c}\text { Number of } \\
\text { populations }\end{array}$ & $5 \%$ & $50 \%$ & $95 \%$ & \\
\hline Breast & 33 & 41.6 & 85.1 & 95.6 & 63 \\
Bronchial & 50 & 9.2 & 21.3 & 37.2 & 132 \\
Colon & 50 & 10.8 & 17.0 & 22.7 & 70 \\
Melanoma & 45 & 0.50 & 10.7 & 28.5 & 262 \\
Thyroid & 49 & 4.5 & 10.6 & 24.9 & 52 \\
Non-Hodgkin's & 50 & 3.8 & 7.6 & 10.3 & 86 \\
Pancreatic & 50 & 4.0 & 6.0 & 8.2 & 70 \\
Kidney & 50 & 2.6 & 5.7 & 8.9 & 111 \\
Stomach & 50 & 2.5 & 5.2 & 20.9 & 354 \\
Brain & 50 & 2.2 & 4.6 & 5.7 & 76 \\
Myeloma & 49 & 1.0 & 2.7 & 7.4 & 274 \\
Liver & 50 & 1.5 & 2.5 & 8.3 & 272 \\
Hodgkin's & 45 & 0.40 & 2.1 & 3.1 & 129 \\
Esophageal & 49 & 0.40 & 1.2 & 3.5 & 258 \\
Mouth & 45 & 0.50 & 1.0 & 1.4 & 90 \\
Small intestinal & 45 & 0.50 & 0.90 & 2.0 & 167 \\
Laryngeal & 41 & 0.20 & 0.70 & 1.3 & 157 \\
\hline
\end{tabular}

breast $=-0.740$, stomach versus $\mathrm{HL}=-0.741$, small intestinal versus laryngeal $=0.751$, breast versus mouth $=0.775$, breast versus non- $\mathrm{HL}=0.840$, small intestine versus myeloma $=0.857$, and stomach versus liver $=0.875$.

For each cancer type, the female/male ratio of ASR values was listed and pairwise analyzed as described above. Of the 136 coefficients, 47 were found to be significant. Of these 47 , with 10 greater than \pm 0.600 , and four being highly strongly correlated: pancreatic versus esophageal $=0.718$, pancreatic versus bronchus $=0.724$, pancreatic versus laryngeal $=0.768$, and laryngeal versus bronchus $=0.768$.

For each population, the female/male ratio of ASR values was then listed in order: Mouth, esophageal, stomach, colon, liver, pancreatic, breast, bronchial, melanoma, kidney, brain, thyroid, myeloma, laryngeal, HL, non-HL, and small intestinal. Brazil was omitted from this analysis because fewer than 100 cases were reported for seven cancer types. Correlation coefficients were calculated for the 49 remaining populations in all pairwise combinations. Of these 1,152 correlation coefficients, the lowest was 0.950 (Table IV).

Table V shows percentiles for various comparative statistics between females and males. In each of 24 populations in this study that also reported comparative statistics, life expectancy was found to be longer, and GNI and suicide risk smaller (18), and adult mortality rates smaller (19) in females than males. Of the 24 nations reporting IMR and U5MR, all but China reported lower rates for females than males $(20,21)$. Male babies may receive
Table III. Ratio of female to male age standardized rate (ASR) percentiles and variation about the median (Varmed).

\begin{tabular}{lcccc}
\hline Cancer & \multicolumn{3}{c}{ Ratio percentile } & \multirow{2}{*}{ Ratio Var ${ }_{\text {med }}$} \\
\cline { 2 - 3 } & $5 \%$ & $50 \%$ & $95 \%$ & \\
\hline Breast & 75.1 & 114.4 & 179.3 & 91 \\
Thyroid & 2.6 & 3.2 & 4.4 & 56 \\
Melanoma & 0.67 & 0.89 & 1.2 & 60 \\
Hodgkin's & 0.54 & 0.82 & 1.0 & 56 \\
Colon & 0.56 & 0.75 & 0.88 & 43 \\
Brain & 0.67 & 0.74 & 0.89 & 30 \\
Pancreatic & 0.58 & 0.74 & 0.83 & 34 \\
Small intestinal & 0.54 & 0.71 & 0.83 & 41 \\
Non-Hodgkin's & 0.63 & 0.70 & 0.78 & 21 \\
Myeloma & 0.59 & 0.69 & 0.83 & 35 \\
Mouth & 0.25 & 0.53 & 0.67 & 79 \\
Bronchial & 0.23 & 0.50 & 0.83 & 120 \\
Kidney & 0.38 & 0.50 & 0.57 & 38 \\
Stomach & 0.37 & 0.48 & 0.55 & 38 \\
Liver & 0.27 & 0.33 & 0.48 & 64 \\
Esophageal & 0.08 & 0.22 & 0.42 & 155 \\
Laryngeal & 0.06 & 0.16 & 0.25 & 40 \\
\hline
\end{tabular}

Table IV. Correlation coefficients between male and female age standardized rate (ASR) values across populations in fixed order.

\begin{tabular}{ll}
\hline Cancer & $\mathrm{r}$ \\
\hline Myeloma & 0.99 \\
Stomach & 0.98 \\
Liver & 0.98 \\
Thyroid & 0.98 \\
Non-Hodgkin's & 0.98 \\
Melanoma & 0.97 \\
Kidney & 0.94 \\
Hodgkin's & 0.93 \\
Small intestinal & 0.93 \\
Brain & 0.92 \\
Pancreatic & 0.85 \\
Colon & 0.75 \\
Esophageal & 0.68 \\
Mouth & 0.54 \\
Breast & 0.51 \\
Laryngeal & 0.43 \\
Bronchial & 0.19 \\
\hline
\end{tabular}

better care than female babies in China $(21,22)$. In each reporting population, females were found to smoke less than males (22). In general, females reportedly ate less meat and drank less alcohol than males $(23,24)$. Plasma testosterone is lower in females than males (25). It is hard to escape the conclusion that, from birth onward, females are inherently healthier than males (26). 
Table V. Comparison of male and female statistics.

\begin{tabular}{|c|c|c|c|c|}
\hline \multirow[t]{2}{*}{ Parameter } & & \multicolumn{3}{|c|}{ Percentile } \\
\hline & & $5 \%$ & $50 \%$ & $95 \%$ \\
\hline Life expectancy (in years) & $\mathrm{F} / \mathrm{M}$ & 1.04 & 1.07 & 1.17 \\
\hline Infant mortality rate (deaths per thousand births) & $\mathrm{F} / \mathrm{M}$ & 0.72 & 0.82 & 1.23 \\
\hline Under 5 mortality rate (deaths per thousand births) & $\mathrm{F} / \mathrm{M}$ & 0.747 & 0.818 & 1.17 \\
\hline Adult mortality rate (deaths per thousand) & $\mathrm{F} / \mathrm{M}$ & 0.375 & 0.527 & 0.653 \\
\hline Gross national income (PPP dollars) & $\mathrm{F} / \mathrm{M}$ & 0.415 & 0.624 & 0.794 \\
\hline Suicide rate (deaths per thousand) & $\mathrm{F} / \mathrm{M}$ & 0.153 & 0.280 & 0.518 \\
\hline
\end{tabular}

F: Female; M: male.

\section{Discussion}

It is suspected that females are healthier than males because at any given chronological age, their tissues, except for breast and thyroid, are physiologically younger than male tissues. Four differences in cancer risk warrant discussion: i) between genders, ii) among cancer types, iii) among populations, and iv) across ages.

Between genders. For non-HL, myeloma, and cancer of the mouth, esophagus, stomach, colon, liver, pancreas, bronchus, kidney, and larynx, the ASR was greater for males than females in every population studied. With minor exceptions, the same conclusion applies to HL and cancer of the brain and small intestine. The minor exceptions were as follows: For China Shanghai, the ASR for cancer of the small intestine was the same for both genders. In China Shanghai and China Hangzhou, the ASR for cancer of the brain was greater for females. In Croatia and Greater Poland, the ASR for HL was greater for females, while in Poland Lower Silesia and Ukraine, it was the same in both genders.

For cancer of the breast and thyroid, the ASR was greater for females than males in every population studied. This pattern of greater risk in males than females except for breast and thyroid cancer cannot be attributed to chance or the lifestyle/environment because neither would yield such global consistency. The gender pattern of risk must be attributed to genes that are common in populations across the globe.

Melanoma is conspicuously different, displaying a more varied pattern of gender risk. While most populations exhibit greater melanoma risk for males than females, the exceptions are numerous and notable: Melanoma risk was found to be the same for both genders in China Beijing, China Hong Kong, Japan Osaka, Japan Aichi, and Korea Seoul, and greater for females in Denmark, Norway, Ireland, UK, UK England, UK Scotland, Germany Lower Saxony, Spain Basque Country, and Ukraine. These exceptions suggest that for melanoma, population-specific environmental, lifestyle or genetic factors often overwhelm the cause of the normal gender pattern of risk.

In every population studied, LE was longer, and AMRs, GNI, suicide rates, and age-standardized smoking rates were lower in females than males. Of the 24 nations reporting gender-specific IMR and U5MR, all but China reported lower rates for females. There is a suspicion that the same would be true in China if equal care were given to babies of both genders (20-21). Females eat less meat and drink less alcohol than males (22-24). Plasma testosterone is lower in females than males $(25,26)$. From birth onward, females are healthier than males (27). Any or all of these differences might contribute to the differences in cancer risk between the genders. But height is, arguably, the most conspicuous difference between the genders, and cancer risk is positively correlated with height (28). Unfortunately, reliable data on height in populations across the world are lacking.

The ratio for female/male height in centimeters in the United States is non-Hispanic Black $=0.927$, non-Hispanic Asian $=0.922$, non-Hispanic White $=0.921$, and Hispanic $=0.920$ (29). Height may be a surrogate for tissue size, which may be a surrogate for cancer target size.

Cancer types. Cancer of the bronchus and colon is common, while those of the mouth and small intestine are rare, in both genders in all populations studied (Tables I and II). This is an interesting pattern because essentially every carcinogen that enters the bronchus passes first through the mouth and every carcinogen that enters the colon passes first through the small intestine. What can explain the disparity in risk of cancer between these neighboring tissues? It cannot be chance because the disparity is large and consistent. It cannot be population-specific environment, lifestyle or genes because the disparity is invariant across all populations studied. The disparity must be due to genes that are common across our species but differ in expression between tissues of high and low cancer risk. It is these genes that are the principal cause of cancer. 
In both genders in all populations studied, non-HL as well as cancer of the pancreas, brain, and kidney are less common than cancer of the bronchus and colon, and more common than cancer of the mouth and small intestine, again suggesting that relative cancer risk among tissues is caused by genes that are common across the world but vary in expression among tissues of tumor origin.

Melanoma, again, demonstrates an unusual pattern of relative risk. In both genders in the Asian populations and U.S. Blacks, melanoma is less common than cancer of the mouth and small intestine, and in Australia and New Zealand, more common than cancer of the bronchus and colon. Some other cancer types also demonstrate unusual patterns of relative risk. Stomach cancer is more common than cancer of the bronchus and colon in Japan and Korea, but less common than those types of cancer everywhere else. HL is less common than cancer of the mouth and small intestine in Japan and Korea, and more common than these everywhere else. Thyroid cancer is conspicuously common in Korea, as is myeloma in U.S. Blacks. These unusual patterns suggest that population-specific factors can overwhelm the common cause of relative cancer risk among tissues. But unusual patterns are unusual because in the vast majority of cases there is no evidence for populationspecific carcinogenesis.

In all populations, male breast cancer is rare. In most populations, male breast cancer is rarer than mouth and small intestine cancer. In all populations, female breast cancer is more common than cancer of the bronchus and colon. Size is one obvious explanation, as larger breasts carry higher cancer risk (30). Patients with Klinefelter syndrome have larger breasts and higher breast cancer risk than normal males, but smaller breasts and lower breast cancer risk than normal females (31). Breast cancer risk among populations may correlate with breast size. Unfortunately, reliable data on breast size across populations are lacking.

Thyroid cancer, like breast cancer, was found to be more common in females than males in all populations studied. Again, size is a possible explanation, as the female thyroid grows with the menstrual cycle (32). At the age when risk of female thyroid cancer begins to increase, i.e. 15 years (33), thyroid volume is larger in females than males (34).

Of the cancer types studied, laryngeal cancer is rarest in females, and the ratio of female/male ASR smallest. Again, size is an obvious explanation as laryngeal length and volume are larger for males than females (35).

Except for cancer of the bronchus, esophagus, and breast, $\mathrm{Var}_{\text {med }}$ for the ratio of female/male ASR was substantially less than for the individual gender rates (Tables I-III), suggesting that population-specific influence on ASR is the same for both genders except for this group of cancer types. Correlation coefficients for ASR values between genders across the populations studied confirm this suggestion, i.e. they were high except for cancer of the bronchus, esophagus, and breast (Table IV). Ratios of female/male ASR values for breast cancer are unreliable because male breast cancer rates are so low as to be heavily affected by noise. Risks of bronchial and esophageal cancer are, undoubtedly, related to smoking, and smoking rates vary between genders and among populations (5).

The weak or moderate correlation coefficients suggest a unique cause for each cancer type. The correlation between stomach and liver cancer, and the inverse correlation between stomach cancer and HL may be coincident of Asian culture. That for female breast cancer and non-HL, and for myeloma with cancer of the small intestine are interesting. But even a correlation coefficient of 0.8 can only explain $64 \%$ of the relationship between variables. The conspicuous conclusion is that the vast majority of cancer types do not appear to share a common cause. Except for a slight indication that smoking is related to cancer of the pancreas, bronchus, and larynx, the conspicuous conclusion is that, in the vast majority of cases, ratios of female/male risk do not share a common cause because the correlation coefficients are consistently low or moderate at best.

Population. For each population, the female/male ratio of ASR values was listed in order of cancer: Mouth, esophagus, stomach, colon, liver, pancreas, breast, bronchus, melanoma, kidney, brain, thyroid, myeloma, larynx, HL, non-HL, and small intestine. Correlation coefficients were calculated for the 49 populations in all pairwise combinations. Of these 1152 correlation coefficients, the lowest was 0.950 . From this it could be concluded that the reason for the differences in ratio of gender risk among the cancer types studied is the same in all populations. This reason must be intrinsic to the tissues of tumor origin and not to anything population-specific.

Age. For each cancer type, the difference in ASR between populations measures risk from causes other than aging. For each cancer, the difference in age-specific rates between ages 70 and 30 years measures risk from aging. For melanoma and cancer of the bronchus, colon, rectum, stomach, pancreas, kidney, esophagus, larynx, liver, mouth, small intestine, and breast in populations across the world, the maximum difference in ASR between populations is less than the 90th percentile difference between age-specific rates at ages 70 and 30 years (3). By this measure, aging is more important than the collection of non-aging processes.

For melanoma, and cancer of the bronchus, colon, rectum, stomach, pancreatic, kidney, esophageal, laryngeal, liver, mouth, small intestine, and breast in populations across the world, differences were calculated in age-specific incidence rates between ages 40 and 30, 50 and 40, 60 and 50, 70 and 60, and 80 and 70 years. The difference between ages 50 and 40 years 
correlated best ( $r>0.88)$ with ASR (3). This high correlation coefficient suggests that the transition between ages 40 and 50 from minimal to increasing risk can explain most of the ASR. The differences in age-specific rates between ages 50 and 40 years also correlated, with coefficients greater than 0.88 , with the differences in ASR between genders, races, and ethnicities (3). The magnitude of the initial acceleration of risk predicts total risk. This suggests that the mechanism of initial acceleration of risk is the principal cause of cancer, and the differences in cancer risk between tissues, genders, races, and ethnicities.

Why is cancer more common in one tissue than another, or in one gender or race or ethnicity than another? Because the difference in age-specific rates between ages 50 and 40 years is greater for the tissue, gender, race, or ethnicity where cancer is more common than in the tissue, gender, race, or ethnicity where cancer is less common. What happens between ages 40 and 50 years? Perhaps the cancer target is created, and the size of this target determines the size of the risk. Height is correlated with cancer risk (30). Perhaps height is a surrogate for target size. The incidence of cancer may be higher in men than in women because, in part, they harbor larger targets. The larynx is a good example. The breast and thyroid are exceptions, because they are larger in women than men.

Theory. For all cancer types that originate after infancy, risk changes in three phases: Early phase of low risk at young age, followed by a phase of increasing risk with increasing age, followed by a phase of constant or declining risk with increasing age. The phases might be attributed to speciesspecific genes that control telomere dynamics in stem cells that obey a fixed strategy at rates that vary among tissues and between genders, races, and ethnicities, and that can fluctuate in response to environmental and lifestyle factors (36). In the early low-risk phase, tumor-suppressor genes and protooncogenes are protected from mutation, perhaps by the absence of stem cell renewal. In the subsequent phase of growing risk, they become vulnerable to mutation, perhaps due to stem cell renewal. The rate of renewal varies among tissues and between genders, races, and ethnicities, and in response to environmental and lifestyle agents. For any given tissue type, the larger the size, the greater the chance of a carcinogenic mutation. Beyond some tissue-specific age, senescence diminishes renewal and this causes risk to plateau or decline.

\section{Conflicts of Interest}

There is no conflict of interest.

\section{Acknowledgements}

Bartlomiej Kolcz provided valuable help with calculations, and Bevin Rainwater for editing.

\section{References}

1 Dix D: On the role of aging in cancer incidence. J Gerontol Biol Sci 44: 10-18, 1989. PMID: 7374187.

2 Dix D: On the causes of cancer: The argument for inheritance. Int J Health Sci 3(3): 1-6, 2015. DOI: 10.15640/ijhs.v3n3a1

3 Marshall B and Dix D: Human carcinogenesis: Toward a unified theory. Open Access Library J 4: e3707, 2017. DOI: 10.4236/oalib.1103707

4 Clemmesen $\mathrm{J}$ and Busk T: The age distribution of malignant disease in Denmark 1942-44. Acta Radiologica 30: 9-16, 1948. DOI: $10.1177 / 028418514803000102$

5 Armitage P and Doll R: A two-stage theory of carcinogenesis in relation to the age distribution of human cancer. Brit Cancer 11(2): 161-109, 1957. PMID: 13460138.

6 Nolen S, Evans M, Fischer A, Corrada M, Dawas C and Bota D: Cancer-incidence, prevalence and mortality in the oldest-old. A comprehensive review. Mech Ageing Dev 164: 113-126, 2017. PMID: 28502820. DOI: 10.1016/j.mad.2017.05.002

7 Harding C, Pompei F and Wilson R: Peak and decline in cancer incidence, mortality, and prevalence at old ages. Cancer 118: 1371-86, 2012. PMID: 21953606. DOI: 10.1002/cncr.26376

8 Stevens RG, Moolgavkar SH and Lee JA: Temporal trends in breast cancer. Am J Epidemiol 115(5): 759-777, 1982. PMID: 6979244. DOI: 10.1093/oxfordjournals.aje.a113358

9 Volpe E and Dix D: On the role of aging in cancer incidence: Cohort analysis of the lung cancer data. Anticancer Res 6: 14171420, 1986. PMID: 3813495.

10 Tomasetti C and Vogelstein B: Variation in cancer risk among tissues can be explained by the number of stem cell divisions. Science 347: 78-81, 2015. PMID: 25554788.

$11 \mathrm{Wu} \mathrm{S}$, Powers S, Zhu W and Hannun YA: Substantial contribution of extrinsic risk factors to cancer development. Nature 529: 43-47, 2016. PMID: 26675728.

12 Leipold S, Madeyanda A and Dix D: On the role of aging in carcinogenesis. Anticancer Res 21: 4189-4194, 2001. PMID: 11911317.

13 Dix D: On the role of genes relative to the environment in carcinogenesis. Mech Ageing Dev 124: 323-332, 2003. PMID: 12663130.

14 Nejako A, Aranton B and Dix D: Carcinogenesis: A cellular model for the age-dependence. Anticancer Res 25: 1385-1390, 2005. PMID: 15865095.

15 Mucci LA, Hjelmborg JB, Harris JR, Czene K, Havelick DJ, Scheike T, Graff RE, Holst K, Möller S, Unger RH, McIntosh C, Nuttall E, Brandt I, Penney KL, Hartman M, Kraft P, Parmigiani G, Christensen K, Koskenvuo M, Holm NV15, Heikkilä K, Pukkala E, Skytthe A, Adami HO and Kaprio J: Familial risk and heritability of cancer among twins in Nordic countries. JAMA 315: 68-76, 2016. PMID: 26746459.

$16 \mathrm{Kim}$ SK: Common aging pathways in worms, flies, mice and humans. J Exp Biol 210: 1607-1612, 2007. PMID: 17449826. DOI: $10.1242 /$ jeb004887

17 Bray F, Colomet M, Mery L, Pineros M, Znaor A, Zanetti R and Ferlay J (eds): Cancer Incidence in Five Continents, Volume XI, summary tables by site (ICD-10 three-digit rubrics), Lyon: International Agency for Research on Cancer, 2017. Available from: http://ci5.iarc.fr

18 Malik K: Human Development Report, 2014, U.N. Human Development Programme, N.Y., Tables 5 and 11. Available at: http://hdr.undp.org/en/content/human-development-report-2014 
19 Klugman J: Human Development Report, 2010, U.N. Human Development Programme, N.Y., Table 14. Available at: http://hdr.undp.org/en/content/human-development-report-2010

20 U.N. Department of Economic and Social Affairs/Population Division (2011) Sex Differential in Childhood Mortality, United Nations Publication ST/ESA/SER.A/314. Available at: https:// www.un.org/en/development/desa/population/publications/mortal ity/sexdifferentials.asp

21 Sawyer C: Child mortality estimation: Estimating sex differences in childhood mortality since the 1970s. PLoS Med 9(8): e1001287, 2012. PMID: 22952433. DOI 10.1371/journal. pmed.1001287

22 WHO Report on the Global Tobacco Epidemic: The MPOWER package. Geneva WHO, 2008. Available at: https://www.who.int/ tobacco/mpower/2008/en

23 Love HJ and Sulikowski D: Of meat and men: Sex differences in implicit and explicit attitudes toward meat. Front Psychol 9: 559, 2018. PMID: 29731733. DOI: 10.3389/fpsyg.2018.00559

24 CDC. Alcohol fact sheet. Available at: https://www.cdc.gov/ alcohol/fact-sheets/mens-health.htm

25 Warraich HJ and Califf RM: Differences in health outcomes between men and women: Biological, behavioral, and societal factors. Clin Chem 65(1): 19-23, 2019. PMID: 287334.

26 Crimmins E, Shim H, Zhang YS and Kim JK: Differences between men and women in mortality and the health dimensions of the morbidity process. Clin Chem 65(1): 135-145, 2019. PMID: 30478135.

27 Dix D: The female health-survival advantage: Paradox unwarranted. Int J Public Health 59: 213, 2014. PMID: 23974353. DOI: 10.1007/s00038-013-0505-y

28 Nunney L: Size matters: height, cell number and a person's risk of cancer. Proc Biol Sci 285(1889): pii: 20181743, 2018. PMID: 30355711. DOI: $10.1098 / \mathrm{rspb} .2018 .1743$

29 Fryar C, Gu Q, Ogden C and Flegal K: Anthropometric reference data for children and adults: United States, 2011-2014. Vital Health Stat (39): 1-46, 2016. PMID: 28437242
30 Jansen LA, Backstein RM and Brown MH: Breast size and breast cancer: A systematic review. J Plastic Reconstr Aesth Surg 67: 1615-1623, 2014. PMID: 25456291. DOI: 10.1016/j.bjps.2014. 10.001

31 Brinton LA: Breast cancer risk among patients with Klinefelter syndrome. Acta Paediatrica 100: 814-818, 2011. PMID: 21241366.

32 Fister P, Gaberscek S, Zaletel K, Gersak K and Hojker S: Thyroid volume changes during pregnancy and after delivery in an iodine-sufficient Republic of Slovenia. European J Obstet Gyn Reproductive Biol 145: 45-48, 2009. PMID: 19406558. DOI: $10.1016 /$ j.ejogrb.2009.03.022

33 New York State Cancer Registry: Cancer Incidence and Mortality in New York State, 1976-2016. Available at: https://www. health.ny.gov/statistics/cancer/registry/table 6/tbgthyroidqueens.htm (age at diagnosis/death, thyroid cancer, Queens County).

34 Lamichhane T, Pant S, Lamichhane B, Gautam C, Paudel S, Yadav $\mathrm{B}$ and Lamichhane H: Age- and gender-specific changes in thyroid size and thyroid function test values of euthyroid subjects. J Biosci Med 6(11): 59-73, 2018. DOI: 10.4236/jbm.2018.611007

35 Inamoto Y, Saitoh E, Okada S, Kagaya H, Shibata S, Baba M, Onogi K, Hashimoto S, Katada K, Wattanapan P and Palmer JB: Anatomy of the larynx and pharynx: Effects of age, gender and height revealed by multidetector computed tomography. J Oral Rehabil 42(9): 670-677, 2015. PMID: 25892610. DOI: 10.1111/joor.12296

36 Monaghan P and Ozanne S: Somatic growth and telomere dynamics in vertebrates: relationships, mechanisms and consequences. Phil Trans Roy Soc B 373(1741): pii: 20160446 , 2018. PMID: 29335370. DOI: 10.1098/rstb.2016.0446 